\title{
Virtual Environment Modeling for Battery Management System
}

\author{
Chang-hao Piao*, Qi-fan Yu*, Chong-xi Duan*, Ling Su** and Yan Zhang ${ }^{\dagger}$
}

\begin{abstract}
The offline verification of state of charge estimation, power estimation, fault diagnosis and emergency control of battery management system (BMS) is one of the key technologies in the field of electric vehicle battery system. It is difficult to test and verify the battery management system software in the early stage, especially for algorithms such as system state estimation, emergency control and so on. This article carried out the virtual environment modeling for verification of battery management system. According to the input/output parameters of battery management system, virtual environment is determined to run the battery management system. With the integration of the developed BMS model and the external model, the virtual environment model has been established for battery management system in the vehicle's working environment. Through the virtual environment model, the effectiveness of software algorithm of BMS was verified, such as battery state parameters estimation, power estimation, fault diagnosis, charge and discharge management, etc.
\end{abstract}

Keywords: Battery management system, Virtual environment, Electric vehicle, Simulation

\section{Introduction}

With the global energy shortage and the requirements of environmental protection, it is a big challenge to solve the problems of energy shortage and environment pollution. Because new energy vehicles (including pure electric vehicles, hybrid electric vehicles and fuel cell car) can satisfy the demand for low emission and low fuel consumption, new energy automobile has become an important trend in the future automotive industry $[1,2]$. The power battery system is one of the key parts in the field of electric vehicle, which has appealed universal attention and research among the major automobile manufacturers and research institutions.

As is known to all, if the battery could work properly, it must be with a battery management system. Therefore, battery management system is a prerequisite for the battery system, which is a critical module of new energy vehicles. The battery management system has a lot of functions, such as real-time collection of battery system parameters, SOC estimation, fault diagnosis and alarm, consistency compensation of the battery, etc [3, 4].

New energy vehicles have many problems in the present, such as short driving range, short life, high cost and poor safety etc. And these problems are due to the limitations of power battery system in performance, durability, cost and safety, so power battery system has become the bottleneck

$\dagger \quad$ Corresponding Author: Institution of Pattern Recognition and Application, Chongqing University of Posts and Telecommunications China. (Zhangyan@cqupt.edu.cn)

* Institution of Pattern Recognition and Application, Chongqing University of Posts and Telecommunications Chongqing 400065, China.

** Chongqing Changan New Energy Automobile CO., LTD., Chongqing 401120 China.

Received: April 24, 2013; Accepted: March 28, 2014 of the industrialization of electric vehicles [5]. In view of the importance of the battery and battery management system, researchers have done a lot of research both power battery model and the battery management system software. The established battery model mainly includes equivalent circuit model, simplified electrochemical model and the neural network model [6]. Based on the actual function of battery management system, battery management system model has developed [7]. But we have not found the virtual environment model for battery management system in the domestic and foreign public documents. The virtual environment model for battery management system gave a new idea to test the validity and reliability between battery management software and power battery in different properties, and also the matching degree between vehicle and power battery system under different conditions. This paper put forward the virtual environment model for battery management system for the first time, which was used to test the feasibility and effectiveness of battery management system software in the early stage of the design, greatly improving the reliability of battery management system software.

\section{Structure of Virtual Environment}

The virtual environment model for battery management system was used as the core of battery management system software. The overall virtual environment model is a facing-forward simulation model of new energy vehicles. First, according to the input and output parameters of battery management system software, the battery model and vehicle control unit model were determined, which were directly connected to battery management system software model. The input and output parameters of battery 
management system software model mainly included current, voltage, temperature, SOC, SOH, charge and discharge power, grade of fault diagnosis, etc. Among them, battery model provided some input parameters for battery management system software, such as current, voltage and temperature. The output parameters of battery management system software such as SOC, balance control signal, charge / discharge power and grade of fault diagnosis were sent to the vehicle control unit model. Using the received information, the vehicle control unit model sent the relay control command to battery management system. According to the command and the actual working condition of battery system, battery management system controlled the switch of positive relay, negative relay, and the recharge relay to realize the corresponding control. The other output parameters of battery management system software, such as the open circuit voltage, the internal resistance, state-of-health, were used for general reference, which were important to test battery performance. The input/output parameters and their connectivity of the battery management system software model are shown in Fig. 1.

Based on the input / output requirement of battery management system, this paper constructed the virtual environment model for battery management system under vehicle environment. The overall model mainly included driving cycle model, driver model, vehicle control unit model, battery management system software model, battery model, power system model, wheel model, etc. Among them, the power system model, wheel model and vehicle dynamics model which were the basic models [8]. The focus we studied is battery management system software model, battery model, vehicle control unit model, driver model, etc. By integrating vehicle model and controller model resource, we linked the modules above together, formed the closed loop feedback of vehicle speed, and

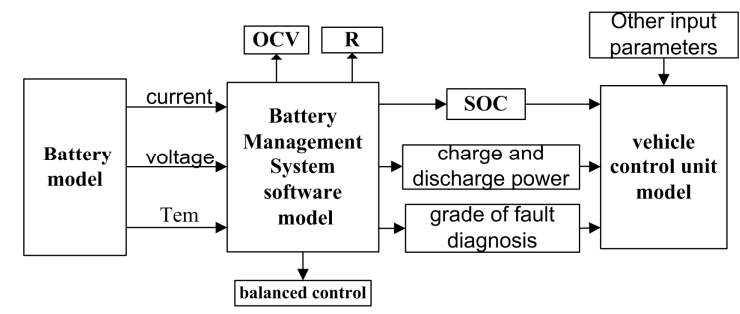

Fig. 1. Input and output parameters of battery management system software

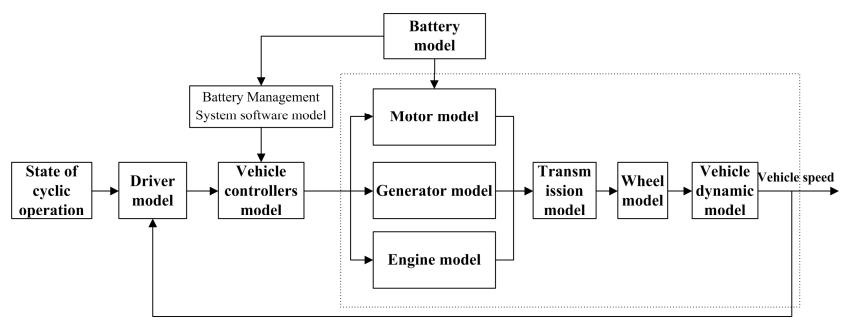

Fig. 2. The overall structure of the virtual environment model for battery management system established facing-forward simulation model of new energy vehicles, and completed the virtual environment model for battery management system under vehicle environment. The overall structure of the virtual environment model is shown in Fig. 2.

\section{System Modeling}

\subsection{Battery management system software model}

The battery management system software model can realize the function of battery management system, which plays an important role in actual running of power battery. The proposed battery management system software model can fulfill the estimation of battery parameters, and then output control signal and error alarm signal. The battery management system software has all functions of BMS, such as SOC estimation, open circuit voltage estimation, internal resistance estimation, charge and discharge power estimation, $\mathrm{SOH}$ estimation, grade of fault diagnosis, balanced control, etc, as shown in the Fig. 3. The battery management system software model can quickly complete the offline validation on the algorithm of battery management system. This software could replace the real hardware development board to some extent, which has an important meaning in the development of power battery system.

Battery management system software model consists of an input / output processing module, SOC estimation module, open-circuit voltage and internal resistance estimation module, charge and discharge power module, fault diagnosis module, balanced control module, up / down electric module and $\mathrm{SOH}$ estimation module. These modules are, respectively, related to the functions above, which are expected to be accomplished according to the detail descriptions as follows. Of course, these modules and their functions are in service of steady and good performance of power battery.

1) SOC estimation module: according to multiple sensor data fusion technology, state of charge could be estimate [7].

2) Charge and discharge power module: based on the

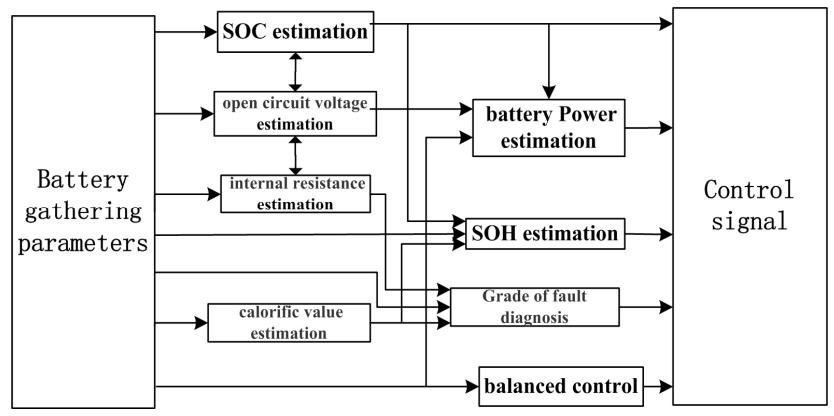

Fig. 3. Overall design of the battery management system software model 
battery system current total voltage, SOC and temperature, real-time online estimation of the maximum charge and discharge power of Ni-MH battery could be realized with the lookup table algorithm.

3) Balanced module: with battery parameters being detected and analyzed, to open or close of balance circuit would be controlled, and then the battery system would be kept consistency in the best state.

4) up/down electric module: according to the command of vehicle control unit, battery management system controls the switch of positive relay, negative relay, and the pre-charge relay to realize up/down electric of high voltage system.

5) Open-circuit voltage and internal resistance estimation module based on the statistical method were to realize the online estimation [9].

6) Fault diagnosis module: the battery management system detected cell voltage, module voltage, charge/ discharge current, temperature and battery insulation; then, the module would calculated the battery failure level, and finally a control command would be given by the battery system.

7) $\mathrm{SOH}$ estimation module: this paper proposes an online calculation method of battery system life to estimate current state of health of the battery system.

\subsection{Battery model}

Battery system has nonlinear characteristics, and it is hard to describe power battery accurately. Based on Thevenin model $[10,11]$, a linear regression analysis method was applied to identify key parameters of the battery model, which could update model parameters with statistics in real time. The nonlinear time-varying battery model is shown in Fig. 4.

In order to confirm whether the battery model could truly reflect the real characteristics of battery with higher accuracy and reliability, some experiments and simulations were carried out. Ni-MH battery for electric vehicle, which consisted of five $1.2 \mathrm{~V} / 6 \mathrm{Ah}$ unit cells, was used to acquire test data. While, simulation data were from the battery model under the same conditions with the experiments'.

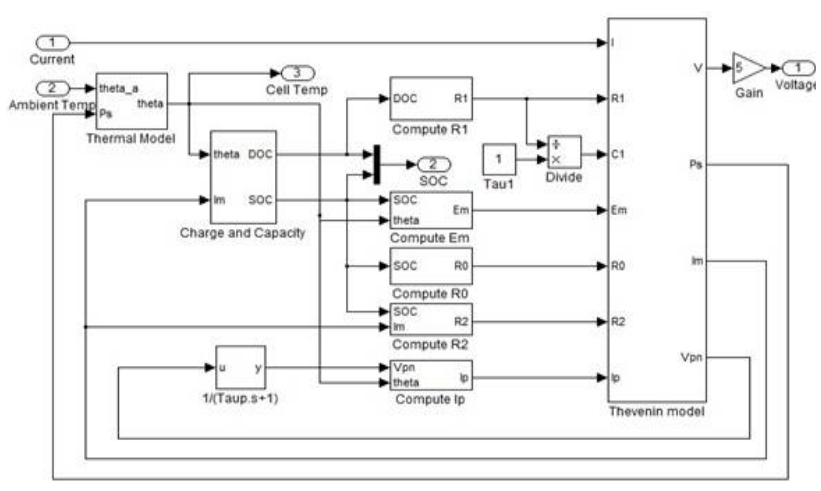

Fig. 4. The battery model
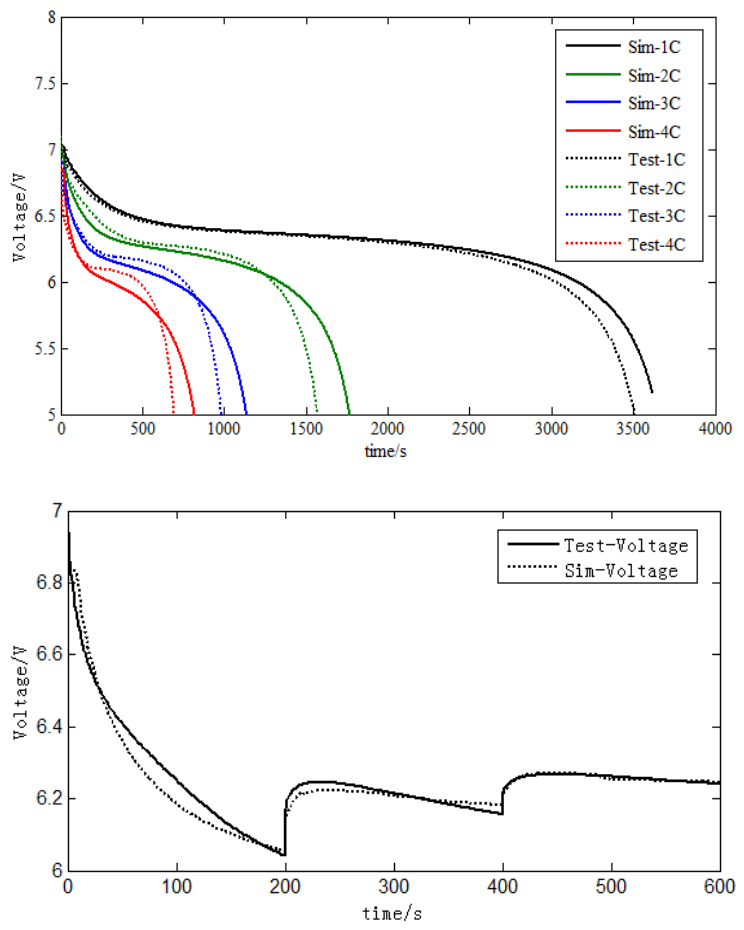

Fig. 5. The curves of battery discharge of experiments and simulation

Under different discharge rates (1C, 2C, 3C, 4C), constant current discharge and variable current discharge experiments were carried on. By comparison, the result turned out to be reasonable and reliable. The curves of test data and simulation data are shown in Fig. 5.

Some essential parameters of battery model, such as temperature and SOC, were from corresponding calculation model as follows

1) Internal resistive losses and ambient temperature contribute to battery temperature or electrolyte temperature. A first order differential Eq. (1) below was modeled to estimate the change in battery temperature.

$$
\theta(t)=\theta_{\text {int }}+\int_{0}^{t} \frac{\left(P_{s}-\frac{\left(\theta-\theta_{a}\right)}{R_{\theta}}\right)}{C_{\theta}} d \tau
$$

Where, $\theta$ is the battery's temperature in ${ }^{\circ} \mathrm{C} ; \theta_{a}$ is the ambient temperature in ${ }^{\circ} \mathrm{C} ; \theta_{\text {int }}$ is the battery's initial temperature in ${ }^{\circ} \mathrm{C}$, assumed to be equal to the surrounding ambient temperature; $P_{S}$ is the $I^{2} R$ power loss of $R_{0}$ and $R_{2}$ in Watts; $R_{\theta}$ is the thermal resistance in ${ }^{\circ} \mathrm{C} /$ Watts; $C_{\theta}$ is the thermal capacitance in Joules $/{ }^{\circ} \mathrm{C}, \tau$ is an integration time variable, $t$ is the simulation time in seconds

2) An ampere-hour integration approach was applied to calculate SOC. The detail computing formulas are shown Eq s. (2-3) 


$$
\begin{gathered}
S O C=\frac{C_{c d}}{C_{e}} \times 100 \% \\
S O C_{k}=S O C_{0}-\int_{0}^{t_{k}} \frac{\eta I d t}{C_{e}}
\end{gathered}
$$

Where, $C_{c d}$ is the current quantity of electric charge; $C_{e}$ is nominal capacity of battery; $I$ is current; $\eta$ is the charge-discharge efficiency; $S O C_{0}$ is the initial value of battery.

\subsection{Vehicle control unit model}

Based on the control strategy of logic gate, the vehicle control unit model was established. Energy management and distribution strategy was presented on the basis of SOC balance. First of all, a set of parameters, and limited the working range of engine, electric motor, battery, and other key parts needed to be set up. According to the preset rules, the hybrid system operating mode could be judged and chose in the actual operation [12-14]; and by setting different parameter value for simulation, a group of optimal parameter values were finally determined.

When the battery SOC has a large fluctuation range in the normal operation of vehicles, the strategy could do power compensation according to the actual situation of vehicle and real-time state of battery. The battery SOC can keep balance from a to $\mathrm{z}$ in circulation condition. When the vehicle has a large speed and high power demand, battery system can provide extra power to meet the power demand of the vehicle.

The input parameters of the vehicle control unit model mainly included accelerator pedal instruction, brake instruction, car speed, motor speed, ICE speed, SOC, maximum charge / discharge power of battery, grade of fault diagnosis, and so on. The output parameters were engine on/off signal, engine torque signal, motor torque signal, battery fault alarm signal, etc. The diagram of the vehicle control unit model is shown in Fig. 6.

The process of the vehicle control unit model is shown in Fig. 7. In order to check the current state of the battery, the battery fault detection should be done first. If the

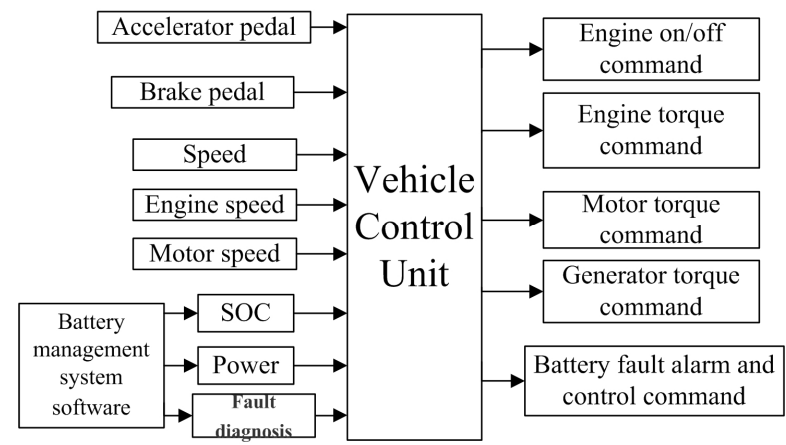

Fig. 6. The diagram of the vehicle control unit model.

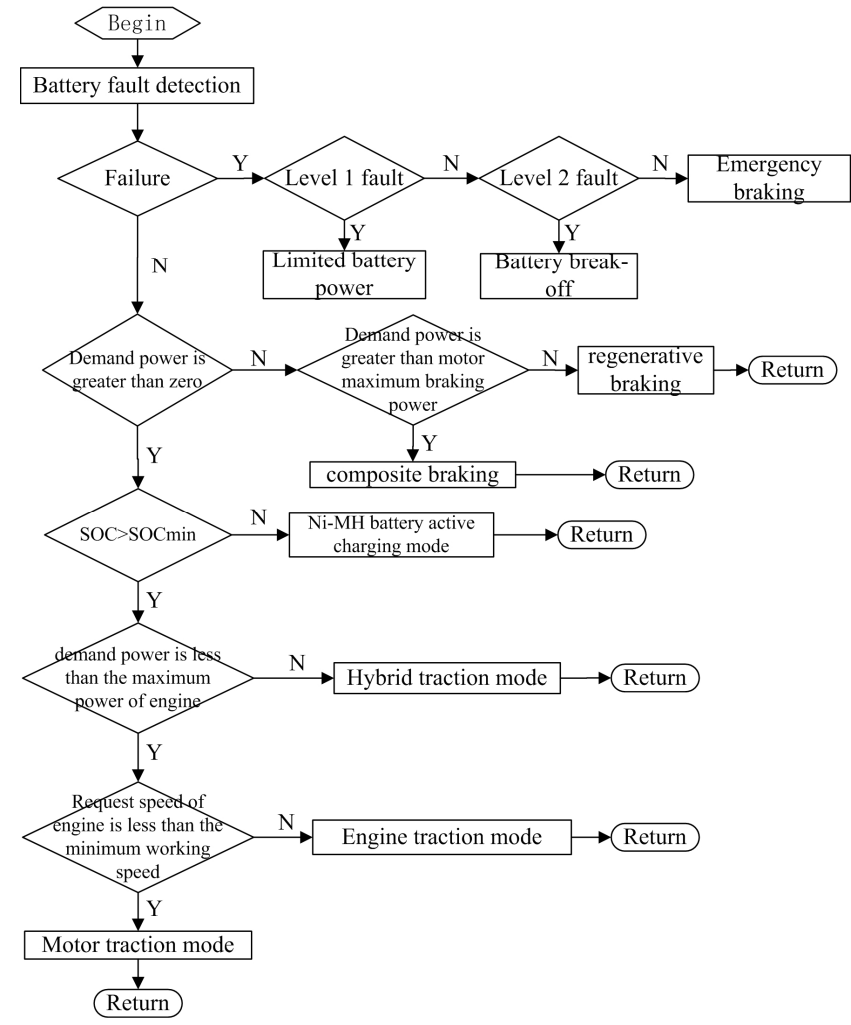

Fig. 7. The process of the vehicle control unit model

battery is failure, the failure would be dealt with; then, if the demand power of vehicle is greater than zero, the battery SOC would be considered whether it is lower than the limited value. If so it is, the battery needs to be charged by the engine, or the value of required power should be taken into account. Otherwise, the vehicle will enter the corresponding operation process of brake; if the value of demand power is greater than the maximum power of engine, the vehicle could use hybrid traction mode, or else the request speed of engine needs to be judged if it is less than the minimum working speed; if it is less, the vehicle would use engine traction mode, otherwise the motor traction mode would be applied.

According to the control strategy and control rules, the vehicle control unit model was established, and the control strategy was turned into algorithm module. In the vehicle simulation platform, constant test of model and adjustment of the relevant parameters with respect to control rules are based on the simulation result. Finally, we determined a set of optimal parameter value. The control strategy meets both the driver's power demand and the premise of fuel economy with respect to the energy management and distribution strategy on the basis of SOC balance.

\subsection{Driver model}

PID control strategy is usually used in the traditional driver model, but it is difficult to achieve good control 


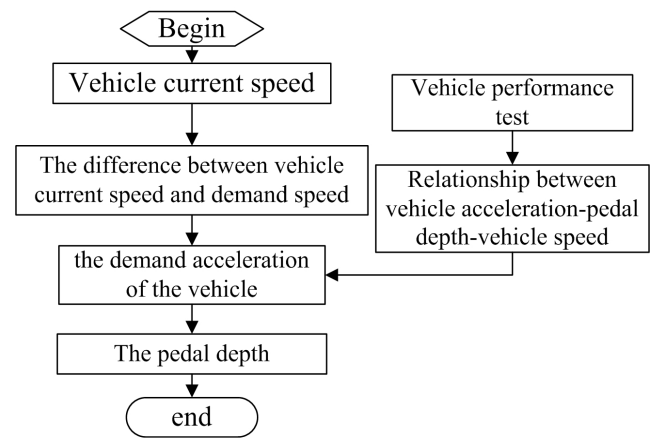

Fig. 8. The diagram of the driver mode

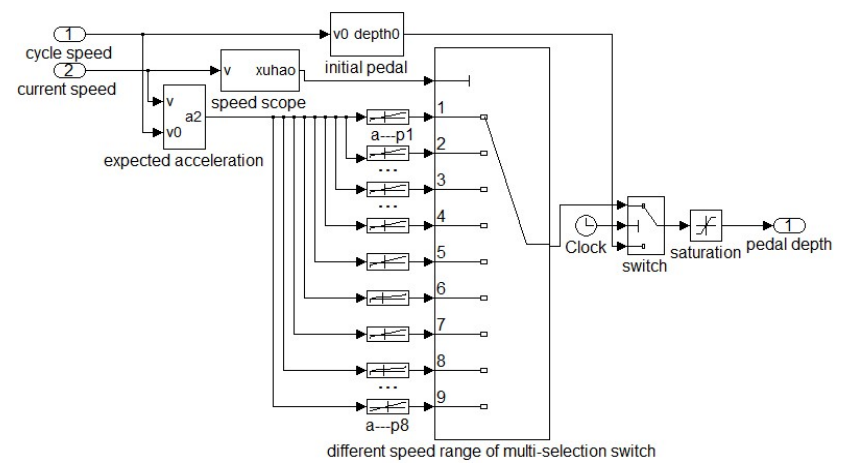

Fig. 9. The top block diagram of driver model

effect for nonlinear and time-varying complex system. It is also very hard to confirm each parameter of PID controller $[15,16]$. This article adopted the driver's following behavior to describe the driver model. A vehicle performance test would be carried out, after which the acceleration of the vehicle by simulating different speed and pedal depth came, with respect to vehicle acceleration-pedal depth-vehicle speed relationship. With the acceleration of the second vehicle, the pedal depth could be determined according to the relationship. The diagram of the driver model is shown in Fig. 8.

The acceleration equation of the vehicle is based on regression analysis of experimental data. The equations are described as followings:

$$
\begin{gathered}
\frac{d v_{n+1}(t+\Delta t)}{d t}=A\left[\lambda \Delta v+\mu v_{n}(t)\right] \\
\Delta v=v_{n}(t)-v_{n+1}(t)
\end{gathered}
$$

Where, $v_{n}(t)$ is the speed of the vehicle; $A, \lambda$ and $\mu$ are regression parameters. According to regression analysis of experimental data, the three parameters $\lambda, \mu$ and $A$ are, respectively, $0.586,-0.027,0.913$. The driver model is shown in Fig. 9.

A driver drives a vehicle with the operation of pedal and gear according to different requirements. Once the expected vehicle speed was obtained through the driver model, acceleration would be the derivative of vehicle speed. Given different speed or acceleration with different transmission ration, the relationships of them are as follows [17].

$$
\begin{gathered}
v=\frac{2 \pi}{60} \frac{w_{r} R}{i_{0}} \\
\left(1+i_{g}\right) w_{c}-w_{s}=i_{g} w_{r}
\end{gathered}
$$

Where, $v$ is the vehicle speed, and $w_{c}(\mathrm{rpm}), w_{s}, w_{r}$ are, respectively, the engine speed, generator speed, motor speed; $i_{g}$ is the ratio of sun gear and ring gear in the transmission box; $i_{0}$ is the transmission ratio including differential ratio; $R$ is the radius of the tire.

\section{Virtual environment model}

On the basis of the above model, we integrated the existing vehicle model and controller model resource with the related model, and constructed the virtual environment model which was used offline validation for battery management system, and established the virtual environment model for battery management system software under the vehicle environment. The virtual environment model under the vehicle environment mainly includes driving cycle model, driver model, vehicle control unit model, battery management system software model, battery model, power system model, wheel model, etc. The whole virtual environment model for battery management system is shown in Fig. 10.

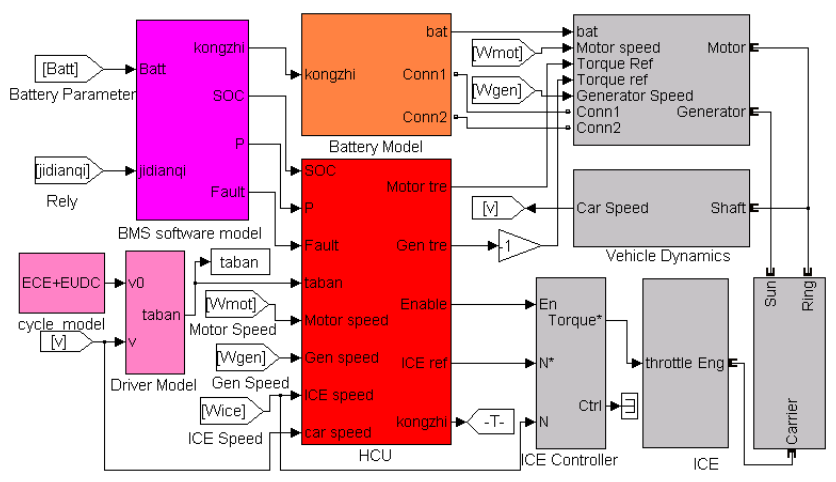

Fig. 10. Virtual environment model for battery management system

\section{Simulation Results}

According to the actual function of the battery management system, and combined with the condition of the vehicle and battery system, the virtual environment model for battery management system software was tested in this paper. Testing is mainly divided into three parts, including standard driving cycle testing, fault diagnosis and treatment testing and limit conditions testing. Combined with the input 


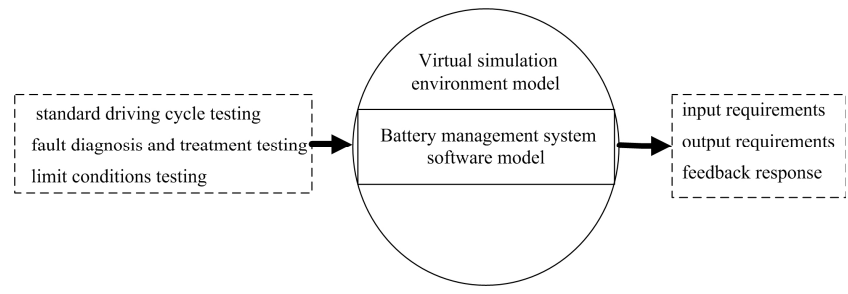

Fig. 11. Test plan of the virtual environment model for battery management system

requirements, output requirements and feedback response of battery management system software model, we observed and analyzed the results. In order to verify whether our battery management system software could achieve the proper functions, we mainly analyzed the performance parameters of power battery system. Combining the functions of the battery management system, we verified whether the battery management system software could receive the needed parameters and achieve proper response. With respect to different modules, simulation results not only play as a role to evaluate the performance of power battery model, but also provide essential information so as to improve the modules.

\subsection{Standard driving cycle testing}

This paper simulated the whole virtual environment model under standard driving cycle (ECE and EUDC) [18, 19]. Fig. 12 represents the curves of battery SOC, current, voltage and temperature in the whole driving cycle. The initial SOC was set at $60 \%$. In the whole driving cycle, the input and output parameters of the battery management system software were within reasonable range. The virtual environment model for battery management system provided the input parameters for battery management system software. The output parameters for battery management system software, such as SOC, charge/discharge power and grade of fault diagnosis were sent to the vehicle control unit model. Then, the vehicle control unit model sent the relay control command to battery management system software, and battery management system would control the switch of positive relay, negative relay, and the precharge relay. With the optimal control strategy of vehicle, the SOC of battery could be maintained at a relatively stable location in the whole cycle. The fluctuation range of SOC declined from $12 \%$ to $8 \%$, the SOC was steady in a certain degree. At the same time, if the battery was abnormal, the battery management system software could accurately detect the failure cause and calculate the fault diagnosis level, then made the corresponding control command to the battery.

After a piece of the driving cycle(the first 200s), including acceleration and deceleration conditions, enlarge it, then especially focused on the change of the parameters such as the current, voltage, temperature and SOC of the battery. As shown in Fig. 12, when the required power of
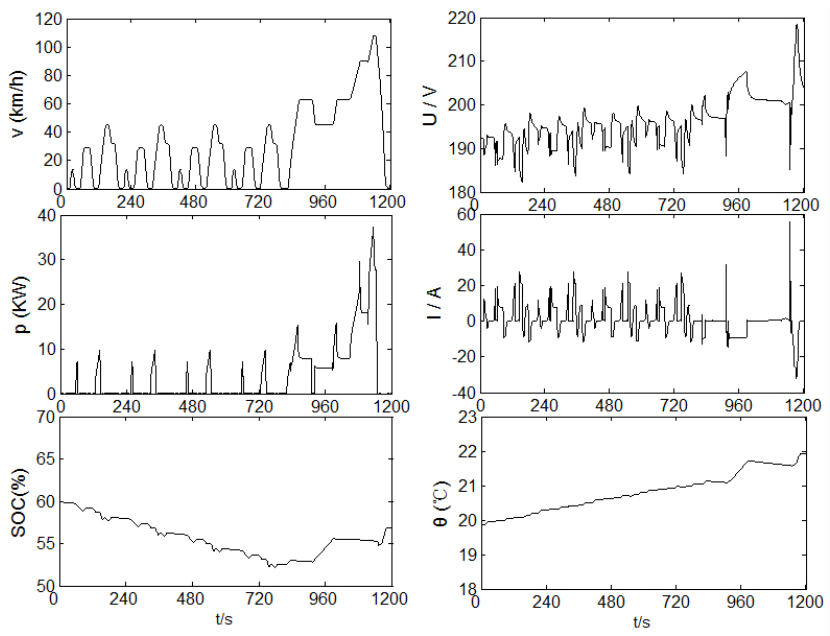

Fig. 12. In the whole driving cycle, the curves of performance parameters of power battery
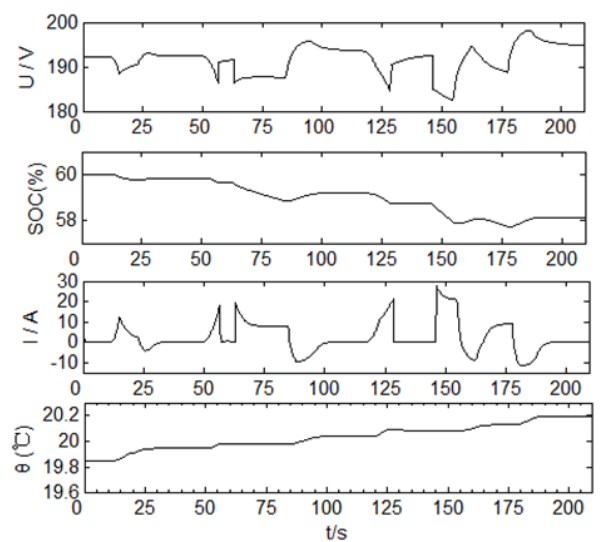

Fig. 13. The first 200s in the driving cycle, the curves of performance parameters of the battery

vehicle was small (as illustrated in Fig. 12, 60s-75s), the battery provide power to the vehicle individually, and the battery was discharged; the current was greater than 0 , and the voltage dropped; the SOC declined, and the temperature of the battery fell slightly. When the vehicle was on regenerative braking mode (as illustrated in Fig. 13, $84 \mathrm{~s}-96 \mathrm{~s}$ ), the battery was charged, and the current was less than 0; the voltage increased; the SOC increased, and the temperature of the battery rose. That is consistent with the actual state of the battery.

Moreover, under standard driving cycle (ECE and EUDC), the virtual environment model testing reflected not only the battery model performance but also its effects on how the driver operated the vehicle and how the worked as expected. The following curves Fig. 14 show the acceleration pedal, transmission ratio, motor torque/speed and engine torque / speed.

\subsection{Fault diagnosis and treatment testing}

Based on the importance of the fault diagnosis of power battery, fault diagnosis and treatment testing was shown in 

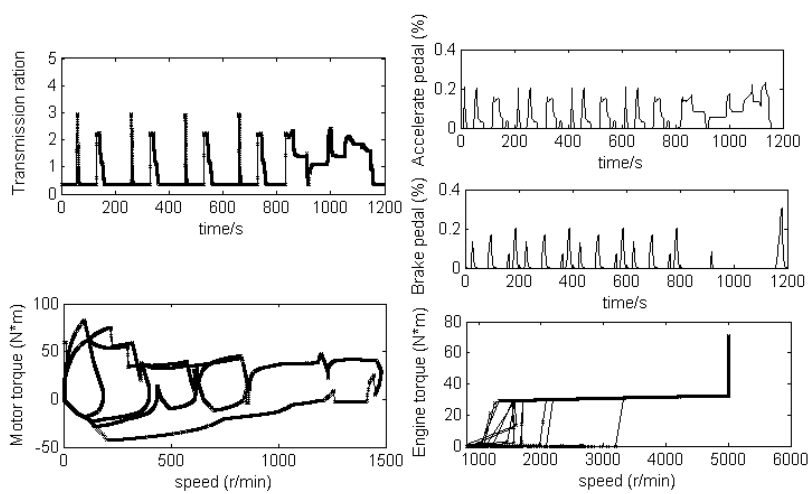

Fig. 14. The curves of accelerate pedal, transmission ratio, motor torque / speed, engine torque/speed
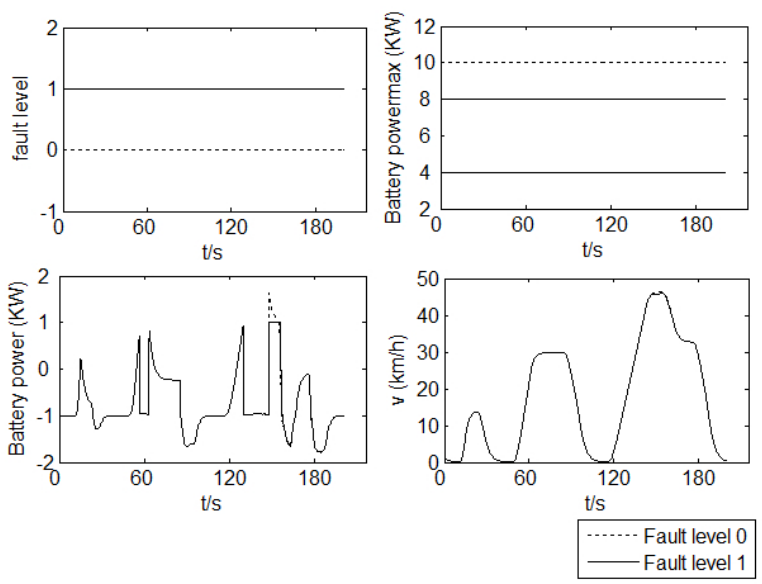

Fig. 15. In the ECE cycle, fault level 1 test of power battery system

this paper. This article first carried on the level 1 of fault test, choosing temperature fault as the source of the power battery. Battery management system software received level 1 fault instruction, and then sent instructions to the vehicle control unit. The vehicle control unit received the information and limited the maximum charging and discharging power of battery system. Through limiting the power, it improved the security of the battery system in abnormal state. Under the ECE working condition, test result of battery system fault level was shown in Fig. 13. When the battery fault level occurred, the battery management system software displayed the fault level and limited the maximum charge and discharge power of battery system.

The level 2 of fault test was similar with the level 1 of fault test. The level 2 of fault test also chose temperature fault as the source of the power battery. Battery management system software received level 2 fault instruction, and then sent instructions to the vehicle control unit. The vehicle control unit received the information and sent a relay disconnection instruction, and then the power battery stopped working. Under the ECE working condition, test result of battery system fault is shown in Fig. 15. When the
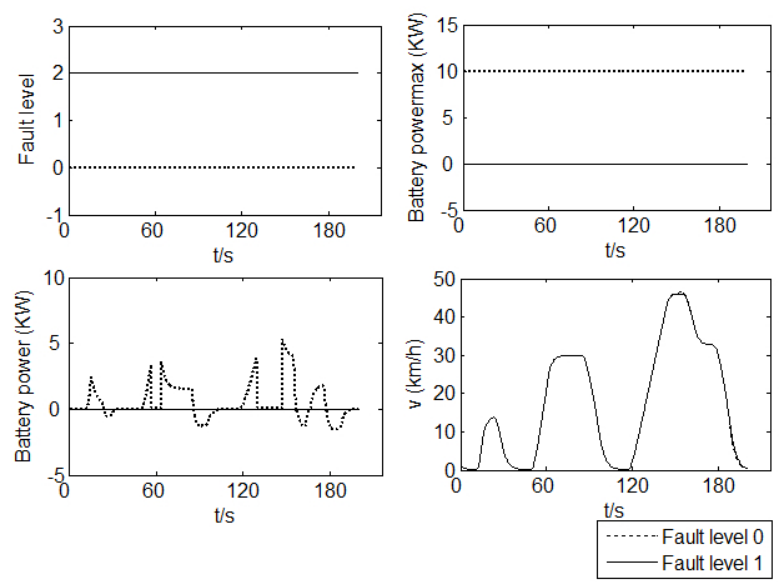

Fig. 16. In the ECE cycle, fault level 2 test of power battery system

battery level fault occurred, the battery management system software displayed the fault level and cut off the relay to make battery stopped working.

\subsection{Limit conditions testing}

Traditional vehicle testing in extreme condition and emergency condition is very difficult [20]. The virtual environment model parameters can be modified by the simulation environment, and got a lot of test data of limit condition. So, it is very convenient to analyze the security and reliability of the power battery and management system and the simulation performance makes it possible to improve the models [21, 22]. Limit condition such as high/low temperature test, high/low pressure test have been reflected in the fault diagnosis test, and the limit condition test focused on the limit of SOC. Limit condition is divided into low SOC and high SOC.

In the low condition test of battery SOC, the initial SOC was set at $1 \%$. We chose standard driving cycle conditions $(\mathrm{ECE}+\mathrm{EUDC})$ to test in the limit conditions. We focused on charge and discharge results of battery state and the vehicle running status. Test results shows that the engine can charge for nickel metal hydride battery, and the battery voltage and SOC all increased in the case of the battery SOC is extremely low under the condition of meeting the driver's power demand. At the same time, cooperated with regenerative braking power generation mode, it also can charge nickel metal battery. When the battery SOC rose to the target value of SOC (50\%), battery system went back to the normal working condition. By observing the actual speed, we found the vehicle running state is normal throughout the working condition, because the battery in the hybrid system is not the only source of power system. Even at very low battery SOC case, it only gives the driver low battery alarm information, but do not affect the normal driving of the vehicle. Test result of the low condition of battery SOC is shown in Fig. 17. 


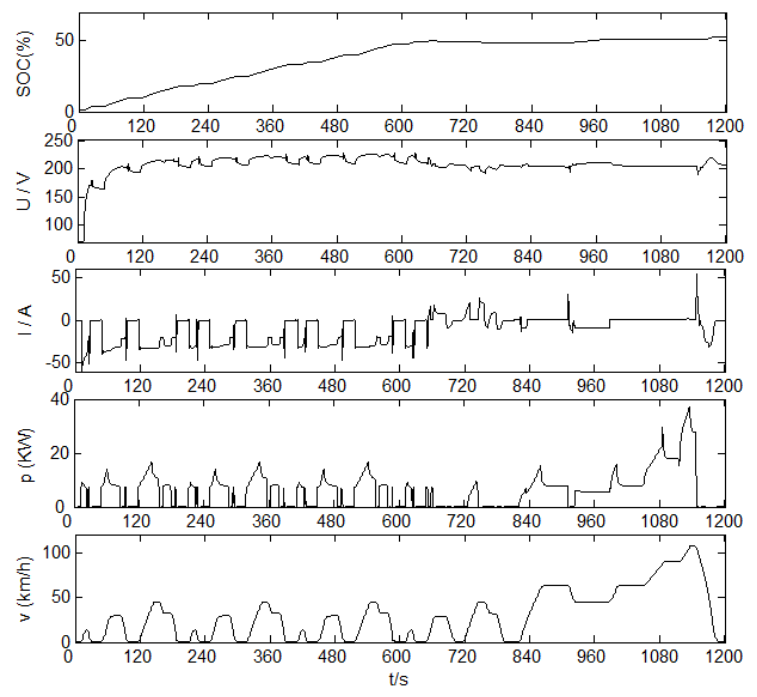

Fig. 17. Test results of the low condition of battery SOC

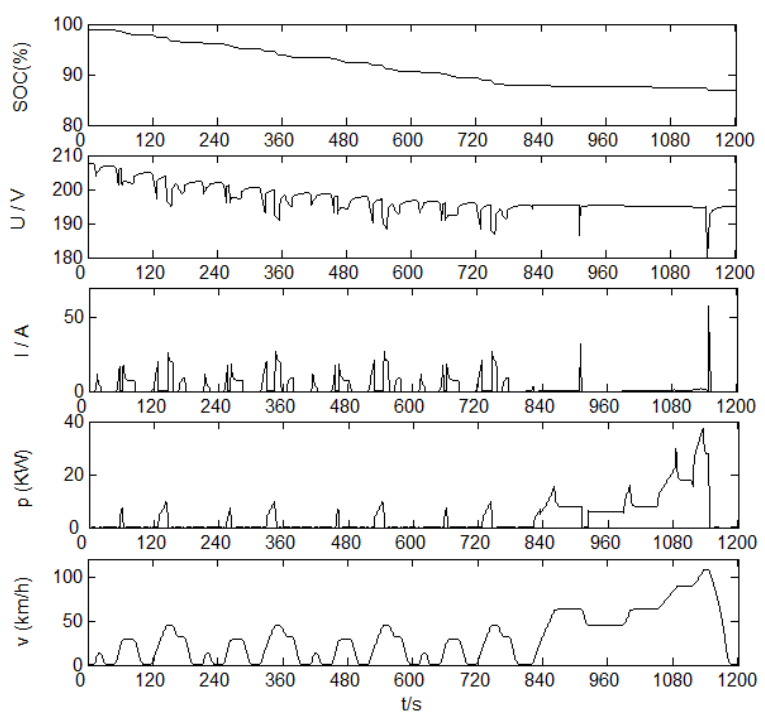

Fig. 18. Test results of the high condition of battery SOC

The test of high condition of battery SOC is similar with the low condition of battery SOC. Initial SOC of battery was set at $99 \%$.We also chose standard driving cycle conditions (ECE+EUDC) to test in the limit conditions. Test results also focus on charge and discharge results of battery state and the vehicle running status. Test result shows that nickel metal battery is not in charging status in the higher range of battery SOC $(80 \%)$, and the battery is only in discharging operation. Even at high battery SOC case, it only keeps the battery in discharging status, but do not affect the normal driving of the vehicle. Test result of battery high SOC conditions is shown in Fig. 18.

When adjusting or optimizing the algorithm of battery management system software, we observed the relevant input/output parameters of battery management system software to judge the effectiveness of battery management system software. On the whole, the virtual environment model for battery management system provided the input / output parameters for battery management system. The battery was monitored in real time and controlled effectively. The entire battery model and battery management system software model was integrated into the vehicle well, and it achieved the interaction with the vehicle.

\section{Conclusion}

The simulation result shows that the virtual environment model provided the needed virtual working environment for battery management system .The battery management system software model achieved all functions of battery management system. The vehicle control unit model received the output parameters of battery management system software and got proper response. The battery management system software achieved the proper function to maintain the normal operation of the battery system.

The virtual environment model for battery management system was applied to research state migration of power battery system in virtual operation environment. Through this technology, we tested the validity and reliability between battery management software and power battery in different properties, and also the matched degree between the vehicle and power battery system under different conditions. The virtual environment is used to achieve simulation of battery system in the vehicle's working environment for verifying effectiveness of BMS.

\section{Acknowledgements}

This work is supported by CQ CSTC (CSTC2013yykfC 60005, CSTC2013jcyjjq60002, CSTC2013jcsf-jcssX0022, CSTC2011BB4145) and NSFC 11247325.

\section{References}

[1] Hu Bei, "The development of Ni-MH battery," China Metal Bulletin, pp. 18-19, Feb. 2010.

[2] Chang Guofeng, Cheng Leitao, Xu Sichuan and Wang Lina, "Structural Optimization Design of Nickelhydrogen Battery Thermal Management System," Journal of Tongji University, pp. 1518-1520, Nov. 2009.

[3] Zhao Yuzhen and Wang Lihua, "Battery Management System Used for HEV," Private science and technology, pp. 40-41, May. 2010.

[4] Feng Xuyun, "Situation and Development on Hybrid Electric Vehicle Battery (Ni-MH) Management System," China Science and Technology Information, pp. 130-131, August.2008.

[5] Zhang Jianbo, Lu Languang and Li Zhe, "Key technologies and fundamental academic issues for traction battery systems," Automotive Safety and 
Energy, vol. 3, no. 2, pp. 87-104, 2012.

[6] Lu Juxiao, Lin Chengtao and Chen Quanshi, "Comparison study of 3 types of battery models for electrical vehicle," Power Sources, vol. 7, pp. 535-538, 2006.

[7] Fu Wenli, "Research on $\mathrm{Ni} / \mathrm{MH}$ battery software simulation platform," Master Dissertation. Chongqing University of Posts and Telecommunications, 2010.

[8] Hauer K-H, "Analysis tool for fuel cell vehicle hardware and software with an application to fuel economy compariosons of alternative system designs," Ph.D Dissertation. University of California Davis, 2001.

[9] Piao Changhao, Fu Wenli and Lei Gaihui, "Online Parameter Estimation of the Ni-MH Batteries Based on Statistical Methods,"Energies, vol. 3, no. 2, pp. 206-215, 2009.

[10] $\mathrm{Hu}$ Xiao song, Li Shengbo and Peng H, "A comparative study of equivalent circuit models for Li-ion batteries," Journal of Power Sources, vol. 198, pp. 359-367, 2012.

[11] Roscher M A, Bohlen O S and Sauer D U, "Reliable state estimation of multi-cell lithium-ion battery systems," IEEE Trans on Energy Conversion, vol. 26, no. 3, pp. 737-743, 2011.

[12] Kimura A, Abe T, Sasaki S, "Drive force control of a parallel-series hybrid system," JSAE Review, vol. 2, no. 3, pp. 337-341, 1999.

[13] Rahman Z, Butler K L and Ehsani M, "A comparison study between two parallel hybrid control concepts," SAE Paper, pp. 990-994, 2000.

[14] Pu Jinhuan, Yan Juanqi and Zhang Jianwu, "Research on Energy Optimal Management and Control Strategies for Hybrid Electric Vehicles," Master Dissertation. Shanghai Jiao Tong University, 2005.

[15] Wang Xiaoyuan, Zhang Jinglei and Meng Zhaowei, "Artificial neural network (ANN) model for car following simulation of microscopic traffic flow," $J$ Shandong Univ of Tech. vol. 18, no. 4, pp. 1-6, 2004.

[16] L. Gan, X. L. Pan, "A method of moving vehicles tracking applied in traffic environment," Journal of Chongqing University of Posts and Telecommunications (Natural Science Edition), vol. 25, no. 3, pp. 408-411, 2013.

[17] X. Li, S. J. Zhang, N. S. Xu, “characteristic analysis and simulation of new-type continuously variable transmission carried in EV," Journal of Chongqing University of Posts and Telecommunications (Natural Science Edition), vol. 24, no. 1, pp. 109-113, 2012.

[18] He Dawei, "Research on the control strategies and simulation in hybrid vehicle based on CVT," Master Dissertation. Hunan University, 2008.

[19] Changhao Piao, Chongxi Duan, Yusheng Li and Sheng Lu, "Research on The Driver's Following
Behavior Based on Hybrid Electric Vehicle Model," in Proceedings of IEEE ICMEME 2012 Conference, Dalian, China, Oct. 2012.

[20] Karden E, Mauracher P, Schope F, "Electrochemical Modeling of Lead/Acid Batteries under Operating Conditions of electric Vehicles," J. Electrochem. Soc, vol. 64 , no. 1 , pp. 175-180, 1997.

[21] Bergveld H J, Kruijt W S and Notteln P H.L, "Battery Management Systems Design by Modelling," aaKluwer Academic Publishers, Netherlands, 2002.

[22] O'Gorman C C, Ingersoll D and Jungst R G, "Artificial neural network simulation of battery performance," in Proceedings of 31st Annual Hawaii International Conference on System Sciences, Hawaii, USA, 1998.

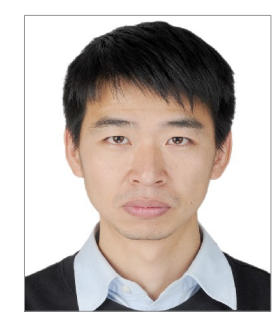

Changhao Piao was born in 1978 in Yanbian (China). He is a Professor in the Chongqing University of Posts and Telecommunications. He received his BS degree from Xi'an Jiaotong University, China in 2011. He obtained Ph.D. in Inha University, Korea. His primary research interests are in new energy vehicles Systems, active safety systems and artificial intelligence.

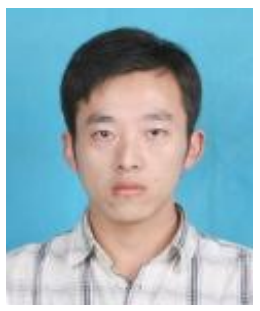

Qifan Yu was born in 1988 in Hubei (China). He is a Postgraduate Researcher in the department of Pattern Recognition and Application at the Chongqing University of Posts and Telecommunications. His research focus was driver's behavior models and vehicle active safety models.

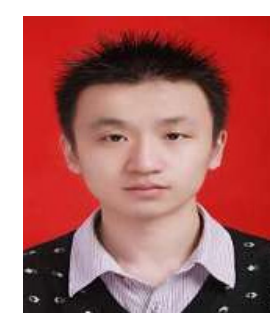

Chongxi Duan was born in 1986 in Shandong (China). He is a Postgraduate Researcher in the department of Pattern Recognition and Application at the Chongqing University of Posts and Telecommunications. His research focus was battery management system model and vehicle control strategy algorithm. 


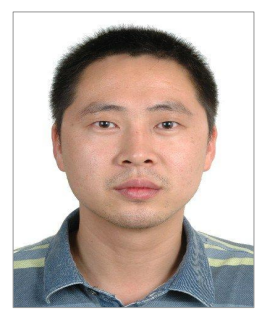

Ling Su was born in 1975 in Chongqing (China). He received his BS degree from Xi'an Jiaotong University, China in 1998 and obtained Ph.D. also from Xi' an Jiaotong University in 2005. $\mathrm{He}$ is now a deputy chief engineer in Chongqing changan new energy automobile co., LTD., His primary research interests are in new energy vehicles and its control system

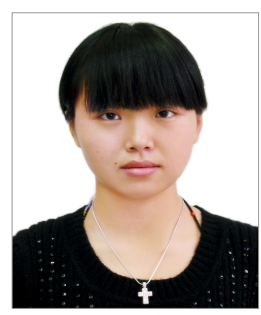

Yan Zhang was born in 1982 in Chongqing (China). In 2006, she received the master degree from the Inha University. Her research focus includes virtural environment modeling. 